\title{
Calculation of the conduction velocity of short nerve fibres
}

\author{
G. H. van der Vliet J. Holsheimer
}

Bio-information group. Department of Electrical Engineering. Twente University of Technology, Enschede, The Netherlands

\section{Bingmann}

Physiological Institute I, University of Münster, BRD

\begin{abstract}
The conduction velocity $v$ of a nerve fibre is calculated from the time delay $\Delta$ of a propagating action potential between two recording sites along the fibre. However, the conventional method of determining $\Delta$ cannot be applied to short nerve fibres. Therefore several linear signal analysis methods for the estimation of $\Delta$ have been compared with regard to the reproducibility of their results obtained from pairs of simultaneous/y recorded action potentials at several small inter-electrode distances. It was found that estimating $\Delta$ from the cross-corre/ogram and as a second one a variant of this method (maximum likelihood time delay estimation) give the most reliable values of $v$ in short nerve fibres.
\end{abstract}

Keywords-Conduction velocity, Time delay, Nerve-fibre, Action potential

\section{Introduction}

THE CONDUCTION velocity of a nerve fibre can be calculated from the time delay of a propagating action potential (a.p.) between two recording sites along the fibre. This is a well known method in clinical and experimental neurophysiology. The time delay between the peaks of two extracellularly and referentially recorded a.p.s can be determined directly, for instance by means of a memory oscilloscope.

In order to obtain a reliable estimate of the conduction velocity, the time delay between the a.p.s has to be large in comparison with the duration of the a.p. (1-2 ms). Normally, the distance between the recording electrodes is at least several centimetres. This method cannot be applied to very short nerve fibres, e.g. the carotid sinus nerve. Under these conditions the two recording electrodes have to be situated only a few millimetres apart and the recorded a.p.s will overlap in time (see Fig. 2). The estimation of the time delay is complicated because in most experiments the a.p.s from one fibre have different waveforms at the two electrodes, which might result from asymmetric recording conditions. Hence, in these short nerve fibres one cannot expect the close relationship between some parameters of the a.p. wave-

First received 22nd August 1979 and in revised form 25th January 1980

$0140-0118 / 80 / 060749+09 \$ 01 \cdot 50 / 0$

(C) IFMBE: 1980 form and the conduction velocity, which has been described in vagal fibres by Paintal (1966).

In order to determine the conduction velocity of a nerve fibre under these experimental conditions we used different functions in the time- and frequency domain. In order to select the best estimator, the time delays calculated from these functions were compared for a series of experiments.

\section{Theoretical aspects}

\subsection{General aspects}

A straight unmyelinated active nerve fibre in a volume conductor has been considered. The electrical properties of the axon are supposed not to change with the length co-ordinate $x$ of the fibre. This means that the shape of the membrane action potential (m.a.p.) is constant and that a.p.s are propagated with a constant velocity $v$.

The relation between the m.a.p. $m$ at $x_{1}$ and $x_{2}$ at a distance $L=x_{2}-x_{1}$ can be given by

$$
m\left(t, x_{2}\right)=m\left(t, x_{1}+L\right)=m\left(t-\frac{L}{v}, x_{1}\right)
$$

$t$ denotes the time variable. The quotient $L / v$ is the time delay $\Delta$ of the propagating m.a.p. between $x_{1}$ and $x_{2}$. So eqn. 1 can be written as

$$
m_{2}(t)=m_{1}(t-\Delta)
$$

Since a.p.s have a limited duration, a value of $T$ can be found, for which the two partly overlapping m.a.p.s $m_{1}(t)$ and $m_{2}(t)$ occur between $t_{0}$ and $t_{0}+T$. So if $m$ 
denotes the deviation from the resting membrane potential, $m_{1}(t)=0$ for $t \leq t_{0}$ and $t \geq t_{0}+T$. This means that $m_{1}$ and $m_{2}$ can be Fourier-transformed:

$$
M_{2}(j \omega)=M_{1}(j \omega) e^{-j \omega \Delta}
$$

For the determination of the conduction velocity $v$, however, we are not recording m.a.p., but extracellular potentials close to an isolated fibre or an active fibre in a small bundle, against a reference electrode (earth) situated at some distance in the volume conducting medium.

The extracellular action potential (e.a.p.), represented by $e(t, x)$, is supposed to be related by a given function to the m.a.p. $m(t, x)$, and is dependent on the position of the recording and reference electrodes with respect to the active nerve fibre (RoSENFALCK, 1969). For two simultaneous measurements near the fibre with respect to a common reference the relationships between $m$ and $e$ can be written in the frequency domain as follows:

$$
\begin{aligned}
& E_{1}(j \omega)=F_{1}(j \omega) M_{1}(j \omega) \\
& E_{2}(j \omega)=F_{2}(j \omega) M_{2}(j \omega)
\end{aligned}
$$

So $F_{1}(j \omega)$ and $F_{2}(j \omega)$ are transfer functions of the respective m.a.p. and e.a.p.

The quotient of the two transfer functions is given by the so-called relation function (defined for the frequency band for which $E_{i}(j \omega) \neq 0$ and $\left.M_{i}(j \omega) \neq 0\right)$ :

$$
F(j \omega)=\frac{F_{2}(j \omega)}{F_{1}(j \omega)}=\alpha(\omega) e^{j \beta(\omega)}
$$

From eqn. 6 we see that this relation function can be divided into two parts: the amplitude ratio function $\alpha(\omega)$ and the difference-in-phase-shift function $\beta(\omega)$.

If the two e.a.p.s have the same shape, then

$$
F(j \omega)=\alpha(\omega)=1 \text { and } \beta(\omega)=0
$$

for all values of $\omega$.

From eqns. 3, 4, 5 and 6, the relationship between the two e.a.p.s in the frequency domain can be written as follows:

$$
E_{2}(j \omega)=E_{1}(j \omega) \alpha(\omega) e^{-j[\omega \Delta-\beta(\omega)]}
$$

Under experimental conditions, however, random noise from the biological preparation and the recording instrumentation, which is assumed to be additive, will influence the measurements. So the recorded signals $r_{i}$ at the electrodes will be composed of the e.a.p. $\left(e_{i}\right)$ and noise $\left(n_{i}\right)$ :

$$
\begin{aligned}
& \underline{r}_{1}(t)=e_{1}(t)+\underline{n}_{1}(t) \\
& \underline{r}_{2}(t)=e_{2}(t)+\underline{n}_{2}(t)
\end{aligned}
$$

The e.a.p. $e_{i}$ is assumed to occur between $t_{0}$ and $t_{0}+T$, so $e_{i}(t)=0$ for $t \leq t_{0}$ and $t \geq t_{0}+T$. $\underline{n}_{i}(t)$ is the realisation of a stochastic (noise) process, which is supposed to be ergodic.
When using a data window $w(t)$, with $w(t)=1$ for $t_{0} \leq t \leq t_{0}+T$ and $w(t)=0$ otherwise, the Fourier transforms of the recorded signals $\underline{r}_{i}(t) w(t)$ are as follows:

$$
\begin{aligned}
& \underline{R}_{1}(j \omega, T)=E_{1}(j \omega)+\underline{N}_{1}(j \omega, T) \\
& \underline{R}_{2}(j \omega, T)=E_{2}(j \omega)+\underline{N}_{2}(j \omega, T)
\end{aligned}
$$

The generation of the recorded signals as described here is summarised in Fig. 1.

Each realisation $\underline{r}_{i}(t) w(t)$ has a noise-signal-ratio function:

$$
\underline{\eta}_{i}(\omega, T)=\frac{\left|\underline{N}_{i}(j \omega, T)\right|}{\left|\underline{E}_{i}(j \omega)\right|}
$$

Now the bandwidth of a recorded action potential (r.a.p.) $\underline{r}_{i}(t) w(t)$ can be defined as the frequency band for which the mean-square value of the noise-signalratio function $m s v\left[\eta_{i}(\omega, T)\right] \leq 1$.

The conduction velocity $v$ of the nerve fibre concerned can be calculated from an estimation of the time delay between the two r.a.p.s $\underline{r}_{1}(t) w(t)$ and $\underline{r}_{2}(t) w(t)$ :

$$
\hat{v}=L / \hat{\Delta}
$$

We may conclude that a discrepancy between the estimated time delay $\hat{\Delta}$ and the real time delay $\Delta$ can be the result of two different processes:

(a) $\beta(\omega) \neq 0$ will result in a systematic error or bias

(b) $m s v\left[\eta_{i}(\omega, T)\right] \neq 0$ will result in a random error.

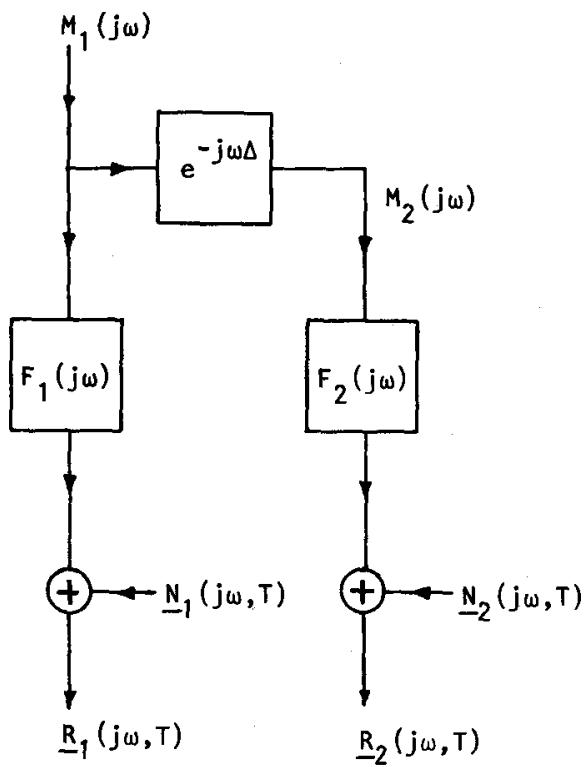

Fig. 1 Schematic representation (in the frequency domain) of the generation of the recorded signals $R_{i}(j \omega, T)$ from the membrane action potential $M_{i}(j \omega)$ at two sites of a nerve fibre and an additive random noise component $\underline{N}_{i}(j \omega, T)$ 


\subsection{Estimation of time delay}

Several methods for calculation of time delay between r.a.p.s are known from the literature (PAINTAL 1966; HUTCHINSON et al., 1970; KraUSE et al., 1972; KALBFLEISCH et al., 1972; HARDY, 1973). These methods result in several estimators for time delay $\hat{\Delta}$, which have been compared in this investigation in order to find which was the most reliable.

In principle, several linear analysis methods for ergodic signals (cf. BENDAT and Piersol, 1971) can be adapted for calculation of the time delay $\Delta$ between two simultaneously recorded r.a.p.s which are considered as two partially deterministic signals. As described in the previous part the choice of the data window $w(t)$ is determined by the position in time of the r.a.p. In the time domain we will use some well defined spots on the r.a.p. waveform $(a)$ as well as the

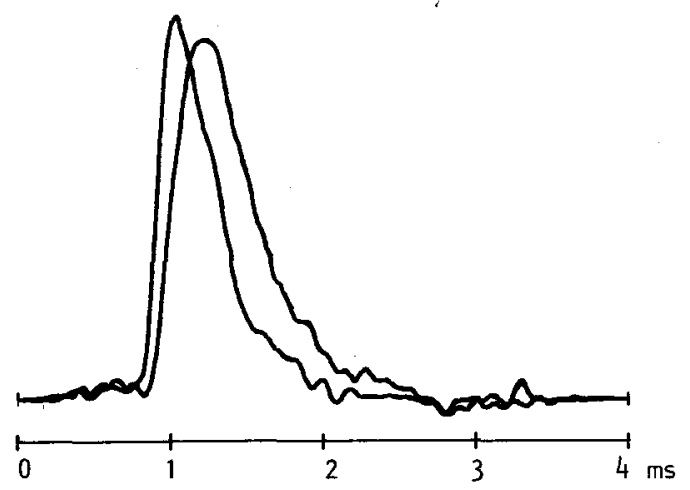

Fig. 2 Pair of simultaneously recorded action potentials from a sinus nerve preparation at $L=2.2 \mathrm{~mm}$, having different waveforms and a time delay

cross correlogram $(c)$; in the frequency domain $\Delta$ will be derived from the phase spectrogram $(b)$. Other methods, which will be discussed here, are calculation of a 'maximum likelihood time delay estimate' for series of pairs of r.a.p.s, according to CARTER $(1976)(d)$ and derivation of $\Delta$ from the energy spectrogram of the differential signal of the two recorded signals (bipolarly recorded action potential), according to the method used in electromyography (LindsTröm and MAGNusson, 1977)(e). The random error as a result of additive noise, related to the estimators of $\Delta$, will be discussed too.

\section{(a) Direct time delay}

The conventional method for estimation of the time delay of a propagating action potential between two recording sites is measuring the time interval between the corresponding peaks of the r.a.p.s. When the shape of the two r.a.p.s is different, time delays estimated in this way can be quite different from the m.a.p. time delay $\Delta$. The systematic error as well as the noise-induced error depend on the choice of the reference spot (peak, half the peak value, first point of inflection). Thereby the systematic error cannot be determined, while the noise-induced error can be estimated from the variability in the time intervals calculated from a number of pairs of r.a.p.s.

\section{(b) Phase time delay}

The phase spectrogram of two e.a.p.s $e_{1}(t)$ and $e_{2}(t)$ is, according to eqn. 7 :

$$
\phi_{E 12}(\omega)=\arg \left[E_{1}(j \omega) / E_{2}(j \omega)\right]=\omega \Delta-\beta(\omega)(14)
$$

After dividing $\phi_{E 12}(\omega, T)$ by the radial frequency $\omega$, we find the phase time delay spectrogram:

$$
\Delta_{p h}(\omega)=\phi_{E 12}(\omega) / \omega=\Delta-\beta(\omega) / \omega
$$

When $\Delta_{p h}(\omega)$ is the estimator of $\Delta$, an unknown systematic error $-\beta(\omega) / \omega$ is made. When the e.a.p.s are affected by additive random noise, the phase spectrogram of $e_{1}(t)$ and $e_{2}(t)$ is estimated from $\underline{r}_{1}(t) w(t)$ and $\underline{r}_{2}(t) w(t)$. Using eqns. 10 and 11 ; we find:

$$
\begin{aligned}
\hat{\phi}_{E 12}(\omega, T) & =\underline{\phi}_{R 12}(\omega, T)=\phi_{E 12}(\omega, T) \\
& +\arg \left[\frac{1+\underline{N}_{1}(j \omega, T) / E_{1}(j \omega)}{1+\underline{N}_{2}(j \omega, T) / E_{2}(j \omega)}\right]
\end{aligned}
$$

So the calculated phase spectrogram $\phi_{R 12}$ is an approximation of the phase spectrogram $\phi_{E 12}$.

In order to calculate the variance $\sigma_{\Delta}^{2}(\omega)$ of the noise-induced error in the estimated phase time delay $\bar{\Delta}(\omega)=\phi_{R 12}(\omega, T) / \omega$ the noise components $n_{1}$ and $n_{2}$ are supposed to have a Gaussian distribution. If in some frequency range $m s v\left[\eta_{1}(\omega, T)\right]$ and $\operatorname{msv}\left[\underline{\eta}_{2}(\omega, T)\right]$ have small values $(<0.02)$, we will find the variance of the noise-induced error:

$$
\sigma_{\Delta}^{2}(\omega) \approx\left\{m s v\left[\underline{\eta}_{1}(\omega, T)\right]+m s v\left[\underline{\eta}_{2}(\omega, T)\right]\right\} / 2 \omega^{2}
$$

The main problem of this method is finding the best frequency band for calculation of the phase time delay as an estimate of the real time delay $\Delta$. It is impossible to correct the calculated time delay for the unknown systematic error $-\beta(\omega) / \omega$. So the only criterion for choosing a frequency band can be the mean-square value of the noise-signal-ratio function in that frequency band of the two r.a.p.s. The condition is that the variance $\sigma_{\Delta}^{2}(\omega)$ of the phase time delay, which can be derived from this function according to eqn. 17 , must be small. $\sigma_{\Delta}^{2}(\omega)$ can be found experimentally from the phase time delay spectrograms of a series of pairs of r.a.p.s. The noise-induced error can be reduced by averaging.

\section{(c) Cross time delay}

Another estimator of the time delay $\Delta$ is the position of the maximum of the cross correlogram $C_{e_{12}}$ of the two e.a.p.s:

$$
C_{e 12}(\tau, T)=\int_{t_{0}}^{t_{0}+T} e_{1}(t) e_{2}(t+\tau) d t
$$


From eqn. 18 it can be calculated that $C_{e 12}(\tau, T)=$ $f(\tau) C_{e 11}(\tau-\Delta, T)$. So the cross correlogram is the convolution of the inverse Fourier transform of the relation function $F(j \omega)$ with the autocorrelogram $C_{e 11}(\tau, T)$ shifted over $\Delta$. The cross correlogram $C_{e 12}(\tau, T)$ has its maximum at $\tau=\Delta_{c}$. If the two e.a.p.s have the same shape $[F(j \omega)=1]$ the cross time delay $\Delta_{c}$ is an unbiased estimator of the m.a.p. time delay $\Delta$.

When $F(j \omega) \neq 1$, we have to take into account a systematic error in the estimated time delay. We calculated that $\Delta_{c}$ is equal to a weighed average of the phase time delay spectrogram of the two e.a.p.s, so the systematic error $\Delta_{c}-\Delta$ is a weighed average of $-\beta(\omega) / \omega$. The weighing function was found to be proportional to the product of the amplitude spectra $\left|E_{1}(j \omega)\right|\left|E_{2}(j \omega)\right|$ and the squared frequency. However, since $\beta(\omega)$ is not known, we cannot calculate $\Delta_{c}-\Delta$.

When the e.a.p.s are affected by additive random noise, then $C_{e 12}(\tau, T)$ is approximated by the cross correlogram of $\underline{r}_{1}(t) w(t)$ and $\underline{r}_{2}(t) w(t)$. So in determining the cross time delay $\Delta_{c}$ a noise-induced error is made, which was found to be the weighed average of $\left[\phi_{R 12}(\omega, T)-\phi_{E 12}(\omega, T)\right] / \omega$. It is rather complex to calculate the variance $\sigma_{\Delta}^{2}$ of the noise-induced error from $\eta_{1}$ and $\eta_{2}$, but this value can be found experimentally. The noise-induced error can be reduced by averaging.

\section{(d) Maximum-likelihood time-delay estimate}

A variant of the cross-time-delay method is described by CARTER (1976). By this method the maximum-likelihood estimate of the time delay between the linearly related components of two signals is calculated. After adaptation this method can be applied to a series of pairs of r.a.p.s. The time delay is given by the position of the maximum of:

$$
\begin{aligned}
C_{r 12}^{\prime}(\tau, T)=\frac{1}{2 \pi} & \int_{-\infty}^{\infty} \exp \left[j \hat{\phi}_{E 12}(\omega, T)\right] \\
& \times \frac{\gamma_{R 12}^{2}(\omega, T)}{1-\gamma_{R 12}^{2}(\omega, T)} e^{j \omega \tau} d \omega
\end{aligned}
$$

The first factor behind the integration sign is an estimate of the normalised cross energy spectrogram of $e_{1}(t)$ and $e_{2}(t)$. The second factor is a weighing function derived from the squared coherence function $\gamma_{R 12}^{2}(\omega, T)$, which can also be estimated from this series of pairs of recorded signals $\underline{r}_{i}(t) w(t)$. The time delay to be found by this method is a weighted average of the mean phase time-delay spectrogram. It was calculated that this weighting function is approximately proportional to $1 / \sigma_{\Delta}^{2}(\omega)$ (see eqn. 17).

\section{(e) Energy spectrograms}

It is expected that action potentials recorded from fast conducting nerve fibres contain more high- frequency components than action potentials from slowly conducting fibres.

So it might be possible to establish a relation between the conduction velocity and some parameter of the energy spectrogram of a single r.a.p.

On the other hand the energy spectrogram of a bipolar recording from a nerve fibre rather contains information about the delay of the propagating action potential between the two recording sites, according to LINDSTRÖM and MAGNUSSON (1977). From eqn. 7 it can be calculated that the energy spectrogram $\left|E_{2}(j \omega)-E_{1}(j \omega)\right|^{2}$ of the (noise-free) differential signal from the two electrodes will show minima (dips) for $\omega \Delta-\beta(\omega)=2 k \pi(k=1,2, \ldots)$. So the time delay can be estimated from the "first dipfrequency' $f_{d 1}(k=1)$ :

$$
\hat{\Delta}=\frac{1}{f_{d 1}}=\Delta-\frac{\beta\left(\omega_{d 1}\right)}{\omega_{d 1}}
$$

and/or from $\left(f_{d 2}-f_{d 1}\right)$ etc. Apart from an extra slope error in the energy spectrogram (VAN DER VLIET, 1978) a systematic error $-\beta\left(\omega_{d 1}\right) / \omega_{d 1}$ is made.

In spite of the advantages of a bipolar recording (little recording artefacts), this method can only be used under certain conditions: $f_{d 1}$ has to lie within the frequency band of the r.a.p. as defined before (see eqn. 12 and further).

A comparison of the methods discussed above, leads to the following conclusions:

(i) if the two simultaneously recorded action potentials have exactly the same shape, i.e. there is no systematic error $[\beta(\omega)=0]$ and no noise-induced error $\left[\underline{N}_{i}(j \omega, T)=0\right]$, all methods will give exactly the same result: all estimators will give the real time delay $\Delta$.

(ii) if the two r.a.p.s have different shapes, i.e. the relation function $F(j \omega) \neq 1$ and/or they are are disturbed by additive random noise $\left[\underline{N}_{i}(j \omega, T) \neq 0\right]$, the methods may give different estimates $\hat{\Delta}$ as a result of differences in sensitivity for these two influences.

\section{Material and methods}

\subsection{Experimental procedures}

In order to test different methods for the estimation of the conduction velocity of short nerve fibres, r.a.p.s were measured from single- and few-fibre preparations of the carotid sinus nerve and the vagal nerve. Experiments were carried out on cats anaesthetised with sodium pentobarbitone ( $50 \mathrm{mg}$ per $\mathrm{kg}$ ), paralysed with gallamine triethiodide and ventilated artificially. Body temperature was kept constant at $37^{\circ} \mathrm{C}$. The carotid sinus nerve was cut close to the glossopharyngeal nerve in order to obtain fibre preparations as long as possible $(4-5 \mathrm{~mm})$. All in-vivo fibre preparations were embedded in paraffin oil.

Recordings were made with two platinum wire electrodes (diam. $0.1 \mathrm{~mm}$ ) fitted in a Perspex holder. 
The interelectrode distance $L$ could be varied. A third platinum wire in the same holder was used to fix the cut end of the fibre preparation, which was placed on the bent tips of the recording electrodes. Recordings were made simultaneously from the two electrodes against a common reference electrode, which was situated in the neck muscles near the recording site. From the measurements it could be concluded that usually only action potentials from one fibre were recorded.

High-impedance low-noise f.e.t. preamplifiers with a frequency band of approximately $1-10000 \mathrm{~Hz}$ were used. Within this frequency range no significant difference in phase shift between the two parallel amplification systems could be detected.

\subsection{Signal storage and pre-processing}

The two simultaneously recorded signals were sampled and stored on-line by a PDP-12 computer with 10-bit analogue-to-digital convertors. Each signal was sampled at a rate of 18500 per second. This sampling rate is high enough, since the power of the recorded signals beyond $6 \mathrm{kHz}$ can be neglected (see Fig. 5). The interval between sampling of the two signals was $12.3 \mu \mathrm{s}$. This interval has to be taken into account when calculating the time delay $\Delta$. Recordings of approximately 10 seconds from several preparations were stored on LINC-tape. Afterwards up to 15 pairs of r.a.p.s from each recording were selected for further data processing on a PDP-11/40 computer.

Each time series holding one r.a.p. had a record length of $4 \mathrm{~ms}$ ( 75 samples). The r.a.p.s were superimposed on low-frequency noise. From each time series the low-frequency noise component, approximated by a linear interpolation between the first and the last (75th) sample, was subtracted. This procedure resulted in new $4 \mathrm{~ms}$ time series from which the first and last sample had a value zero. These time series were multiplied with a cosine-taper data window and extended to time series of 128 points by adding sample points with a value zero. The time series obtained this way were used for estimating the time delay $\Delta$ by the methods discussed in the previous part (Section 2).

\subsection{Data processing}

\section{(a) Direct time delay}

Under the experimental conditions mentioned above the time delays $\Delta$ were expected to be in the same range as the sample interval $\left(T_{s}=54 \mu \mathrm{s}\right)$. In order to get an accurate estimation of the time delay, a much better resolution in the time domain was required. This resolution was obtained by interpolation using fast Fourier transforms. After transformation of each r.a.p. time series into the frequency domain the complex Fourier series was extended by inserting (equidistant) frequency points - having a value zero-beyond the original Nyquist frequency.
After inverse Fourier transformation a new time series having smaller intervals was obtained. In our case the resolution in the time domain was increased 16 times, resulting in $3.4 \mu$ s intervals. This interpolation procedure is acceptable since the power of the analogue signal beyond the Nyquist frequency $(9250 \mathrm{~Hz})$ can be neglected. From the interpolated time series of two simultaneously recorded r.a.p.s the time delay between well defined spots of the waveforms (peak value, half the peak value, first point of inflection) could easily be calculated.

\section{(b) Phase time delay}

The discrete phase time delay spectrogram was calculated from the two Fourier transforms of the time series $\underline{r}_{1}(t) w(t)$ and $\underline{r}_{2}(t) w(t)$, according to eqns. 14 and 15 . The resolution in the frequency domain was increased 8 times (to $18 \mathrm{~Hz}$ ) by extending the time series with additional sample points with value zero. By calculating the deviation $\hat{\sigma}_{\Delta}(\omega)$ of the phase time-delay spectrograms of a number of successive pairs of r.a.p.s, the frequency band could be chosen for calculating the phase time delay as an estimator of the m.a.p. time delay $\Delta$ [see Section $2.2(b)$ ].

\section{(c) Cross time delay}

The discrete cross correlogram of the sampled r.a.p. was calculated according to:

$$
C_{r 12}\left(n T_{s}\right)=\sum_{m=0}^{M-1}\left\{r_{1}\left(m T_{s}\right) r_{2}\left[(m-n) T_{s}\right]\right\}
$$

$M$ being the record length of each r.a.p. time series. The cross time delay, with a resolution $T_{s}=54 \mu \mathrm{s}$, is approximately given by the value of $n T_{s}$ corresponding to the maximum value of $C_{r_{12}}$. In order to get a more accurate estimate of the cross time delay, the resolution was increased 16 times by interpolating the discrete cross correlogram, as has been described in a previous part [Section 3.3(a)].

The same result can be obtained by calculation of the discrete cross energy spectrogram of the two time series and inverse Fourier transformation after insertion of frequency points.

\section{(d) Maximum-likelihood time-delay estimate}

The maximum-likelihood time-delay estimates were calculated according to eqn. 19 from series of pairs of r.a.p.s. Therefore the squared coherence functions were estimated in the same way as has been done for ergodic signals (CARTER, 1976). The resolution was increased 16 times by interpolating the resulting time functions. Standard deviations of the maximum-likelihood time-delay estimator were calculated from the results of several groups of some r.a.p. pairs in each series.

\section{(e) Energy spectrogram}

The discrete energy spectrogram of an r.a.p. $r(t) w(t)$ was calculated from the Fourier transform of 
this time series, by squaring the modulus of $\underline{R}(j \omega, T)$. The resolution in the frequency domain was increased 8 times (to $18 \mathrm{~Hz}$ ) by adding sample points with value zero to the original time series.

In order to get an estimate of the bandwidth (see eqn. 12 and further) of an r.a.p., $m s v|\underline{N}(j \omega, T)|$ can be estimated by averaging the energy spectrograms of a number of epochs (length $T$ ) from the noise signal preceding this r.a.p. The bandwidth was found by comparing this averaged noise spectrogram with the energy spectrogram of the r.a.p. The bandwidth can also be estimated by means of the squared coherence function, if a number of pairs of r.a.p. are available. In this case the mean bandwidth of the two series of r.a.p.s is given by the frequency band for which $\gamma_{R 12}^{2}(\omega, T) \geq 0 \cdot 25$.

\section{Results}

In Section 2 it was concluded that all presented methods of determining the conduction velocity will give exactly the same result if the two r.a.p.s have identical waveforms. So calculations on a simulated pair of identical action potentials could be used for testing the data-processing programs. The experimentally obtained pairs of r.a.p.s had different shapes (see Fig. 2). Thus the different time-delay estimates had to be compared with respect to their reliability. Results will be presented from three recordings from the same carotid sinus nerve preparation at interelectrode distances $L=1.1,1.7$ and $2.2 \mathrm{~mm}$. From each recording a series of over 10 pairs of r.a.p.s were used for estimating the time delay $\Delta$ by the different methods.

\section{(a) Direct time delay}

In Fig. 2 the interpolated time series of a pair of r.a.p.s at $L=2.2 \mathrm{~mm}$ are shown. For each pair of r.a.p.s the time delay was calculated from the positions of the peaks (peak time delay), the centres of the rising flanks (flank time delay) and the peaks of the first derivatives (point-of-inflection time delay). From each recording series the means and deviations of the estimated conduction velocities were calculated. The results are shown in Table 1. From these results we see that the three methods give different results at all values of $L$ tested. Thereby the peak propagation velocities are much smaller than the values obtained by the other two methods. This result can be explained by the differences in waveform of the two r.a.p.s, as can be seen in Fig. 2. From Table 1 it can also be seen that $\hat{v}$ obtained from the flank time delays had the smallest deviation at all three values of $L$.

\section{(b) Phase time delay}

In Fig. $3 a$ the average and the deviation curve of $\hat{\Delta}(\omega)$ of 15 pairs of r.a.p.s at $L=2 \cdot 2 \mathrm{~mm}$ are shown. It can be seen that the mean of $\hat{\Delta}(\omega)$ varies with fre-
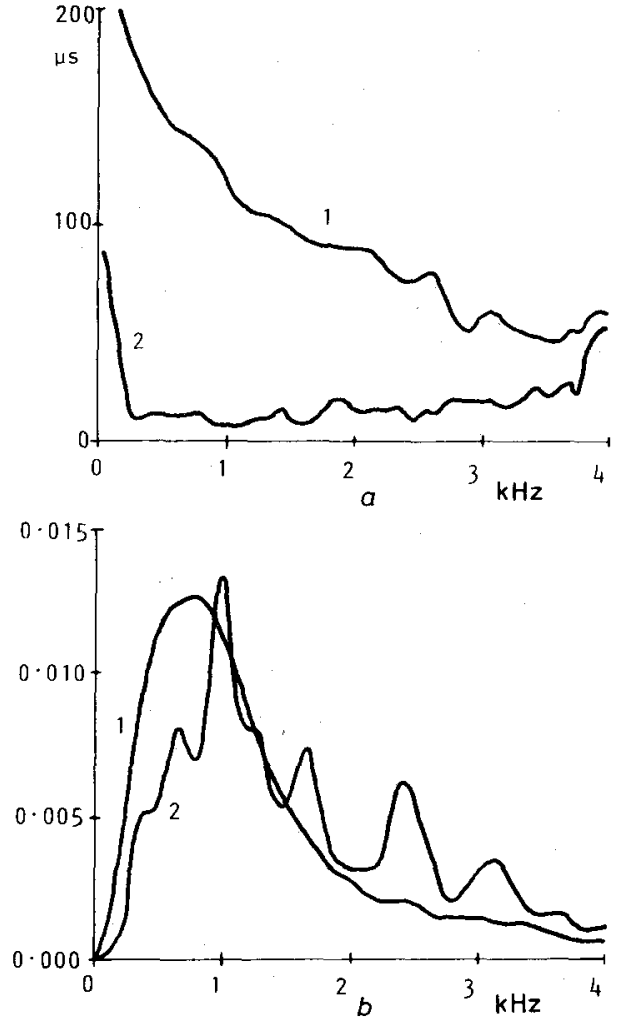

Fig. 3(a) Average phase time delay (1) and deviation (2) as a function of frequency, obtained from 15 pairs of simultaneously recorded action potentials at $L=2.2 \mathrm{~mm}$ from a sinus nerve preparation

(b) Phase-time-delay weighing functions for the cross time delay (I) and for the maximum-likelihood timedelay estimate (2) derived from the same 15 pairs of recorded action potentials

quency. Most other recordings also gave varying mean values. So it was impossible to calculate $\hat{v}$ simply from the average curve. The only criterion for choosing a frequency band for calculating $\hat{\Delta}(\omega)$ was the variance [see Section 2.2(b)]. In Fig. $3 a$ the deviation is relatively small within the frequency range of $300-3800 \mathrm{~Hz}$. In most recordings the frequency range having a relatively small deviation value was restricted to $500-2000 \mathrm{~Hz}$. But also within this frequency range the mean of $\hat{\Delta}(\omega)$ was not constant in most results. In the results presented here the deviation of $\hat{\Delta}(\omega)$ had a minimum at about $1000 \mathrm{~Hz}$. At this frequency-and for comparison at $500 \mathrm{~Hz}$ and at $2000 \mathrm{~Hz}-\hat{v}$ was calculated from the mean of $\hat{\Delta}(\omega)$ (see Table 1). From this Table it can be seen that the values of $\hat{v}$ calculated from $\hat{\Delta}(\omega)$ at $1000 \mathrm{~Hz}$ are only slightly different at the three interelectrode distances. The values of $\hat{v}$ calculated from $\hat{\Delta}(\omega)$ at $500 \mathrm{~Hz}$ and at $2000 \mathrm{~Hz}$ show larger differences at the three values of $L$. 


\section{(c) Cross time delay}

In Fig. $4 a$ the interpolated cross correlogram of a

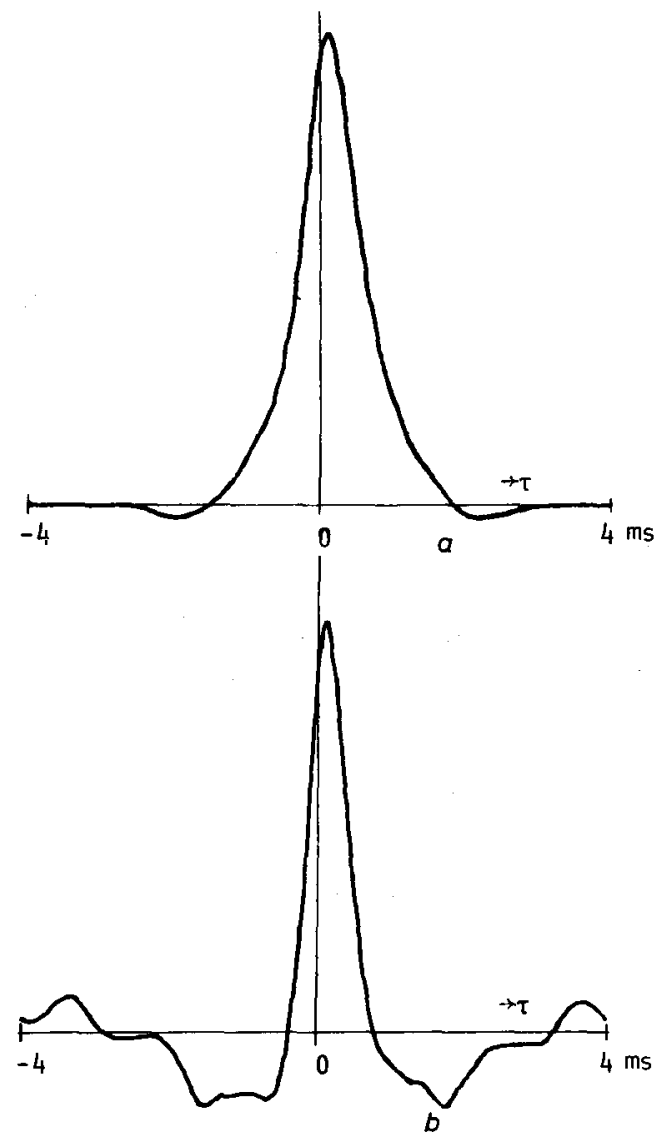

Fig. 4(a) Interpolated cross correlogram of a pair of simultaneously recorded action potentials from a sinus nerve preparation at $L=2.2 \mathrm{~mm}$

(b) Interpolated time function obtained by application of the maximum-likelihood time-delay estimate method on 15 pairs of simultaneously recorded action potentials at $L=2.2 \mathrm{~mm}$ from the same sinus nerve preparation pair of r.a.p.s at $L=2.2 \mathrm{~mm}$ is shown. It can be seen that the position of the maximum differs from $\tau=0$. In Table 1 the mean and deviation of $\hat{v}$ calculated from $\Delta_{c}$ at each value of $L$ are presented. The three mean values are slightly different, but the relatively small deviation areas are amply overlapping.

(d) Maximum-likelihood time-delay estimate

In Fig. $4 b$ the time function as a result of the application of the method of CARTER (1976) to the 15 pairs of r.a.p.s at $L=2.2 \mathrm{~mm}$ is shown. It can be seen that the position of the maximum differs from $\tau=0$. In Table 1 the conduction velocities calculated from the resulting time delays are presented. The three values are slightly different. The deviations were estimated as follows: from different combinations of four pairs of r.a.p.s at one value of $L$, the maximumlikelihood time-delay estimate was calculated. The deviation of the resulting values of $\hat{v}$ was calculated and multiplied by a factor $\sqrt{4}$ in order to allow a comparison with the other deviations from Table 1 (in case $n=4$ ).

In Fig. $3 b$ the (phase time delay) weighing functions for the cross-time delay (1) and for the maximum-likelihood time-delay estimate (2), as described in Sections 2.2(c) and $(d)$, are shown. The two curves were estimated from the same 15 pairs of r.a.p.s at $L=2.2 \mathrm{~mm}$. The two time-delay estimates will be found by multiplication of $\hat{\Delta}(\omega)$ [Fig. $3 a(1)]$ with the corresponding weighing factor at each discrete frequency and adding the resulting values of the whole frequency range $(0-4 \mathrm{kHz})$.

\section{(e) Energy spectra}

The analysis of monopolarly recorded r.a.p.s did not yield clear relations between $v$ and parameters of the energy spectrogram (VAN DER VLIET, 1978). From Fig. 5 it can be seen that the bandwidth of the r.a.p. was limited to approximately $4000 \mathrm{~Hz}$. This value was found for most recordings. This bandwidth is too small for the estimation of $v$ from bipolar recordings as described in Section 2.2(e), when using small values of $L$. The first dip frequency $f_{d 1} \simeq v / L$ to be expected in the energy spectrogram will not be within

Table 1. Average conduction velocities and deviations calculated from different time delay estimates of $n$ pairs of simultaneously recorded action potentials at interelectrode distances $L$ (in $\mathrm{m} / \mathrm{s}$ ). Deviations also in percentage of average values

\begin{tabular}{|c|c|c|c|c|c|c|}
\hline \multirow{2}{*}{$\begin{array}{c}\text { Time delay estimate } \\
\text { (a) (i) peak time delay } \\
\text { (ii) flank time delay } \\
\text { (iii) point of inflection t.d. }\end{array}$} & \multicolumn{2}{|c|}{$\begin{array}{c}L=1.1 \mathrm{~mm} \\
n=13\end{array}$} & \multicolumn{2}{|c|}{$\begin{array}{c}L=1.7 \mathrm{~mm} \\
n=13\end{array}$} & \multicolumn{2}{|c|}{$\begin{array}{c}L=2.2 \mathrm{~mm} \\
n=15\end{array}$} \\
\hline & $\begin{array}{l}15 \cdot 3 \pm 5 \cdot 5 \\
23 \cdot 9 \pm 2 \cdot 6 \\
31 \cdot 4 \pm 15 \cdot 1\end{array}$ & $\begin{array}{l}(36 \%) \\
(11 \%) \\
(48 \%)\end{array}$ & $\begin{array}{l}12 \cdot 0 \pm 2 \cdot 2 \\
23 \cdot 6 \pm 2 \cdot 4 \\
22 \cdot 7 \pm 5 \cdot 4\end{array}$ & $\begin{array}{l}(18 \%) \\
(10 \%) \\
(24 \%)\end{array}$ & $\begin{array}{l}11 \cdot 2 \pm 1 \cdot 9 \\
28 \cdot 2 \pm 1 \cdot 7 \\
39 \cdot 3 \pm 11 \cdot 8\end{array}$ & $\begin{array}{l}(17 \%) \\
(6 \%) \\
(30 \%)\end{array}$ \\
\hline $\begin{array}{l}\text { (b) (i) phase t.d. at } 500 \mathrm{~Hz} \\
\text { (ii) phase t.d. at } 1000 \mathrm{~Hz} \\
\text { (iii) phase t.d. at } 2000 \mathrm{~Hz}\end{array}$ & $\begin{array}{l}15 \cdot 1 \pm 2 \cdot 1 \\
19 \cdot 3 \pm 2 \cdot 7 \\
18 \cdot 6 \pm 5 \cdot 4\end{array}$ & $\begin{array}{l}(14 \%) \\
(14 \%) \\
(29 \%)\end{array}$ & $\begin{array}{l}16 \cdot 2 \pm 1 \cdot 9 \\
17 \cdot 7 \pm 1 \cdot 4 \\
23 \cdot 3 \pm 5 \cdot 1\end{array}$ & $\begin{array}{l}(12 \%) \\
(8 \%) \\
(22 \%)\end{array}$ & $\begin{array}{l}14 \cdot 3 \pm 1 \cdot 1 \\
17 \cdot 9 \pm 1 \cdot 1 \\
24 \cdot 2 \pm 3 \cdot 6\end{array}$ & $\begin{array}{l}(8 \%) \\
(6 \%) \\
(15 \%)\end{array}$ \\
\hline (c) cross time delay & $18 \cdot 0 \pm 1 \cdot 4$ & $(8 \%)$ & $18 \cdot 5 \pm 1 \cdot 3$ & $(7 \%)$ & $18 \cdot 2 \pm 0 \cdot 7$ & $(4 \%)$ \\
\hline (d) max. likelihood t.d.e. & $21 \cdot 1 \pm 3 \cdot 3$ & $(16 \%)$ & $20-6 \pm 3 \cdot 2$ & $(16 \%)$ & $21 \cdot 4 \pm 2 \cdot 9$ & $(14 \%)$ \\
\hline
\end{tabular}


the frequency band of the r.a.p. At $L=3 \mathrm{~mm}$, for example, only values of $v$ smaller than $12 \mathrm{~ms}^{-1}$ can be estimated by this method.

\section{Discussion and conclusions}

From Table 1 it can be seen that the most reproducible results are obtained when applying the cross correlogram or the maximum-likelihood time-delay estimator. Each of these methods gives only small differences between the mean conduction velocities calculated at different interelectrode distances $L$. From Table 1 it can also be seen that the cross-time delay had the weakest sensitivity for additive random noise at all three values of $L$. For all three values of $L$ there is a difference of about $10 \%$ between the values of $\hat{v}$ obtained by these two methods. Since the crosstime delay and the maximum-likelihood time-delay estimate can be considered a weighted average of the phase time-delay spectrogram, this difference is due to a difference between the weighting functions concerned. In the results presented in Fig. $3 b$ the centre of the weighting function determining the maximumlikelihood time-delay estimate (2) is at a higher frequency than the centre of the weighting function determining the cross-time delay (1). Since $\hat{\Delta}(\omega)$ in this experiment decreases with increasing frequency [see Fig. 3a(1)], $\Delta_{c}$ will be the largest one, and thus $\hat{v}$ calculated from $\Delta_{c}$ will be smaller than $\hat{v}$ calculated from the maximum-likelihood time-delay estimate.

The direct time delays calculated for different reference spots, as well as $\hat{\Delta}(\omega)$ calculated at different frequencies, had large deviations and the mean values of $\hat{\Delta}$ obtained at different values of $L$ differed widely. As the energy spectrograms of bipolar r.a.p.s did not yield reliable information about $v$, only the cross-time delay and the maximum-likelihood time-delay estimate turned out to be useful for the determination of $v$ in short nerve fibres. The suitability of both methods, however, depends on the systematic and random errors which should be minimised by the recording and data processing techniques.

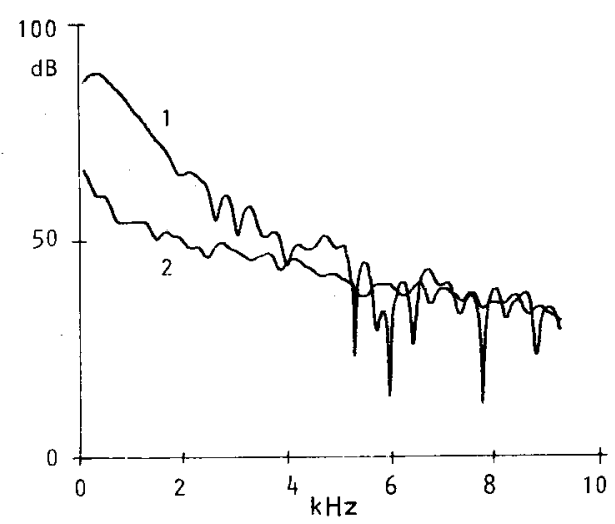

Fig. 5 Energy spectrogram of a monopolarly recorded action potential (1) and the average energy spectrogram of a number of epochs of the preceding noise signal (2)
In Section 2.1 the systematic error was supposed to occur due to a difference between the transfer functions $F_{1}(j \omega)$ and $F_{2}(j \omega)$, as expressed in the relation function $F(j \omega)=\alpha(\omega) e^{j \beta(\omega)}$ eqn. 6. It was found that the effect of an amplitude ratio function $\alpha(\omega) \neq 1$ on $\hat{\Delta}$ is relatively small, in contrast with the effect of a difference-in-phase shift function $\beta(\omega) \neq 0$. From Fig. $3 a$ it can be seen that the systematic error in $\hat{\Delta}=\Delta_{p h}(\omega)$ gets a lower value with increasing frequency. In other experiments the slope of the phase time-delay spectrogram could be quite different, even with a positive instead of a negative slope. So the systematic error varies with the experimental conditions. An important factor will be the difference in position of the recording electrodes with respect to earth (Stein and Pearson, 1971), and, in the case of myelinated fibres, the position of the recording electrodes with respect to the nodes of Ranvier (HUXLEY and STÄMPFLI, 1949; MARKS and LOEB, 1976).

Another possible systematic error source is a difference in m.a.p. waveform at the two electrodes, when the distal electrode is situated too near to the cut end of the nerve fibre. This cut end will cause a change in shape of the m.a.p. near the end, owing to changes in ion gradients across the membrane and to a decrease in resistance between the intra- and extracellular fluid. This possible difference in m.a.p. will be reflected in a difference in e.a.p., and thus in r.a.p. waveforms.

Different shapes of the two m.a.p. might also reflect a difference in local conduction velocity. So the estimated conduction velocity $\hat{v}$ is an average value over the piece of nerve fibre between the electrodes. A systematic error can also be introduced by the recording systems, owing to differences in phase shift of the two systems and crosstalk between them. However, in this series of experiments this type of error can be neglected. Still another type of systematic error can be introduced by the nerve fibre length between the electrodes. $\hat{v}$ is calculated from $\hat{\Delta}$ and $L$ (eqn. 13). $L$ is a good estimate of the fibre length between the electrodes only if the fibre is stretched up to its normal in situ length. So the estimated conduction velocity $\hat{v}=\hat{L} / \hat{\Delta}$.

A possible random error source is the discretisation error in the time domain. After interpolation of the time series the resolution $\Delta t=3 \cdot 4 \mu \mathrm{s}$. Supposing that the difference $\varepsilon$ between the real and the discrete values has a uniform distribution between two discrete moments, then the standard deviation of $\varepsilon$ is $\sigma_{\varepsilon}=\sqrt{(\Delta t)^{2} / 12}=0.98 \mu$ s. This discretisation error is small in comparison with other random errors. For instance, the deviation of the cross-time delay was $>4 \mu \mathrm{s}$ at the three values of $L$. When calculating the direct time delay, the discretisation error is made twice, resulting in $\sigma_{\varepsilon}=1.39 \mu \mathrm{s}$.

In Section 2.1 an unmyelinatec. nerve fibre has been considered. But recordings and calculations were also made of myelinated fibres in which the propagation of m.a.p. is not a continuous process: 
m.a.p.s are only generated at the nodes of Ranvier. However, estimation of $v$ of myelinated fibres is also possible with the methods discussed here, since the e.a.p. waveform at a location between two nodes is a time function in between the two neighbouring nodal e.a.p.s (HuXley and STÄmpFl, 1949; MARKS and LOEB, 1976). Because this internodal e.a.p. is, owing to internodal membrane current, not the result of linear interpolation of the two nodal e.a.p.s, a small systematic error will be introduced. From the results presented in Table 1 -calculated from the cross-time delays and from the maximum-likelihood time-delay estimates-it can be seen that $\hat{v}$ of this probably myelinated fibre is about the same at different values of $L\left(v \simeq 20 \mathrm{~ms}^{-1}\right)$.

FIDONE and SATO (1969) estimated $v$ of myelinated carotid chemoreceptor A fibres directly from the oscilloscope display of two monopolarly, simultaneously recorded a.p.s. They found that $\hat{v}$ ranged from 4 to $53 \mathrm{~ms}^{-1}$, with a median of $16 \mathrm{~ms}^{-1}$ and a semi-interquartile range from 11 to $21 \mathrm{~ms}^{-1}$. The median value is in the same range as the values shown in Table 1. From a comparison of these results, however, no conclusions can be drawn, since it is unknown to what extent the method and the properties of different fibres contribute to the large variability found by FIDONE and SATO. As a whole, the presented results lead to the conclusion that the conduction velocity of short unmyelinated, as well as myelinated, nerve fibres can be estimated reliably via the determination of the cross-time delay and by the calculation of the maximum-likelihood time-delay estimate. Thereby the cross-time delay has the weakest sensitivity for random noise.

\section{References}

Bendat, J. S. and Piersol, A. G. (1971) Random data, analysis and measurement procedures. Wiley \& Sons, New York.
Carter, G. C. (1976) Time delay estimation. Techn. Report 5335, NUSC, New London Laboratory.

Fidone, S. J. and SATo, A. (1969) A study of chemoreceptor and baroreceptor $A$ and $C$ fibres in the cat carotid nerve. J. Physiol., 205, 527-548.

Hardy, W. L. (1973) Propagation speed in myelinated nerve. I. Experimental dependence on external $\mathrm{Na}^{+}$and on temperature. Biophysical J., 13, 1054-1070.

Hutchinson, N. A., Koles, Z. J. and Smith, R. S. (1970) Conduction velocity in myelinated nerve fibres of Xenopus Laevis. J. Physiol., 208, 279-289.

HuXley, A. F. and StämPFLI, R. (1949) Evidence for saltatory conduction in peripheral myelinated nerve fibres. $J$. Physiol. 108, 315-339.

Kalbfleisch, E. W., Olson, D. O. and Halas, E. S. (1972) Bipolar electrode phase shift analysis of multiple unit activity with digital output. Physiol. \& Behav., 9, 873-876.

Krause, D. J., Steadman, J. W. and Williams, Th. W. (1972) Effect of record length on noise-induced error in the cross correlation estimate. IEEE Trans. on Syst. Man and Cybern., SMC-2, 255-261.

LindSTRÖM, L. H. and MAGNusson, R. I. (1977) Interpretation of myoelectric power spectra: a model and its applications. Proc. IEEE, 65, 653-662.

Marks, W. B. and LOEB, G. E. (1976) Action currents, internodal potentials and extracellular records of myelinated mammalian nerve fibers derived from node potentials. Biophysical J., 16, 655-668.

Paintal, A. S. (1966) The influence of diameter of medullated nerve fibres of cats on the rising and falling phases of the spike and its recovery. J. Physiol., 184, 791-811.

Rosenfalck, P. (1969) Intra- and extracellular potential fields of active nerve and muscle fibres. Akademisk Forlag, Copenhagen.

Stein, R. B. and Pearson, K. G. (1971) Predicted amplitude and form of action potentials recorded from unmyelinated nerve fibres. $J$. Theor. Biol, 32, 539-558.

VLIET, G. H. VAN DER (1978) Methoden ter bepaling van de geleidingssnelheid van dunne zenuwvezels. Report no. 182, Bio-information Group, Twente University of Technology, Enschede. 